\section{Achieving antigen-specific immune regulation}

\author{
Kevan C. Herold \\ Naomi Berrie Diabetes Center, Division of Endocrinology and Department of Medicine, \\ College of Physicians and Surgeons, Columbia University, New York, New York, USA
}

\begin{abstract}
A study in this issue of the JCI shows that in response to autoantigens consisting of peptides from normal proteins, patients with diabetes mount a $T$ cell response characterized by production of IFN- $\gamma$ (see the related article beginning on page 451 ). However, in response to these same antigens, $T$ cells from normal control subjects produce IL-10. The antigen-specific response characterized by release of a regulatory cytokine suggests a mechanism for the control of autoimmunity that is initiated at the time of antigen presentation.
\end{abstract}

J. Clin. Invest. 113:346-349 (2004). doi:10.1172/JCI200420963.

For more than a quarter of a century, we have searched for a "trigger" of type 1 diabetes mellitus (T1DM) - an environmental agent or event that initiates the autoimmune response. Epidemiological evidence suggests that one exists (1): the concordance rate of diabetes in identical twins is $<50 \%$; there is seasonal variation in disease presentation, suggesting a viral trigger; and feeding practices, including infant diets, vitamins, and/or breast feeding, appear to modify disease occurrence (2-4). Indeed, one antigen associated with the disease, amino acids 247-279 of glutamic acid decarboxylase-65 (GAD65) shares sequence homology with $\mathrm{P} 2-\mathrm{C}$ protein of coxsackie B, a suggested viral initiator of diabetes (5). In transgenic mice, when the lymphocytic choriomeningitis virus (LCMV) glycoprotein antigen is expressed on $\beta$

\footnotetext{
Address correspondence to: Kevan C. Herold, Naomi Berrie Diabetes Center, Division of Endocrinology and Department of Medicine, Columbia University College of Physicians and Surgeons, 1150 St. Nicholas Avenue, New York, New York 10032, USA. Phone: (212) 305-3171; Fax: (212) 851-5493; E-mail:kh318@columbia.edu.

Conflict of interest: The author has declared that no conflict of interest exists.

Nonstandard abbreviations used: type 1 diabetes mellitus (T1DM); glutamic acid decarboxylase-65 (GAD65); lymphocytic choriomeningitis virus (LCMV); insulinomaassociated-2 (IA-2); regulatory $\mathrm{T}$ cell $(\operatorname{Tr} 1)$; $\mathrm{T}$ cell receptor (TCR).
}

cells, LCMV infection induces autoimmune diabetes (6). Yet despite the continuous search, a foreign agent that is causative has not emerged. Instead, the identified antigens, including GAD65, proinsulin, insulin, insulinoma-associated-2 (IA-2), and others, are normal proteins found in all individuals. One possible explanation for the occurrence of autoimmune responses is that the $T$ cell repertoire of patients with diabetes includes autoreactive clones that have escaped thymic deletion to islet proteins including proinsulin, GAD65, and others.

\section{Models to explain autoimmunity have focused on regulation of peripheral $\mathrm{T}$ cell responses}

One possible explanation for the occurrence of autoimmune responses is that the $T$ cell repertoire of patients with diabetes includes autoreactive clones that have escaped thymic deletion to islet proteins, including proinsulin. These proteins, generally thought to be islet specific, are also expressed in the thymus. In fact, one report has described higher expression of insulin mRNA in the thymuses of individuals with insulin variable numbers of tandem repeats associated with protection from diabetes (7). These investigators postulate that higher insulin expression in the thymuses of disease-protected subjects may lead to deletion of autoreactive cells.
However, animal models, in which antigen expression is limited to islet cells, have not supported the concept that the escape of "forbidden clones" alone is sufficient to account for autoimmunity (8). Peripheral mechanisms of $\mathrm{T}$ cell activation and regulation control autoimmune responses even in the presence of autoreactive $\mathrm{T}$ cells.

Much interest has focused on the phenotype of the $\mathrm{T}$ cell response that differentiates after activation, as most autoimmune $\mathrm{T}$ cell responses are polarized toward a Th 1 phenotype, and Th2 responses are thought to be protective (9). This explanation alone does not account for protection from autoimmunity, because in certain situations, when transferred into an immune-deficient mouse, Th2 cells thought to be protective can cause diabetes (10). In addition, protection requires more than a single Th 2 cell; it takes a "village." When the $\mathrm{T}$ cell repertoire has been restricted experimentally, even conditions that favor Th2 development can result in disease (11). These findings and others from several animal models of T1DM suggest that the differentiation of a large number of cells that are recruited to an inflammatory response toward a nonpathogenic phenotype is an effective means of controlling autoimmune responses. In experimental systems, controlling the phenotype of the effector cell itself may not be sufficient to regulate the response.

A more potent means of regulating immune responses involving regulatory $T$ cells has been studied intensively $(12,13)$. Certain regulatory $\mathrm{T}$ cells, such as $\mathrm{CD} 4^{+} \mathrm{CD} 25^{+} \mathrm{T}$ cells, are present constitutively, whereas others, including a subpopulation of regulatory $\mathrm{T}$ cells ( $\operatorname{Tr} 1$ cells) that produce IL-10 and IFN- $\gamma$, as well as subpopulations of $\mathrm{CD}^{+}$cells including those reactive with class Ib MHC molecules and others that express low levels of CD28, are induced following cell activation $(14,15)$. These cells appear to be very potent in their actions. Ratios of regulatory:effector $\mathrm{CD}^{+} \mathrm{T}$ cells as low as 1:20 have been found to inhibit antigen-specific responses. 


\section{IL-10 as a mediator of $\mathrm{T}$ cell regulation}

A frequent finding among many but not all regulatory $\mathrm{T}$ cells is the production of IL-10 (16). IL-10 can inhibit Th1 and Th2 responses, as well as the maturation of antigen-presenting dendritic cells, suggesting a central role for this cytokine in immune regulation. CD4 or CD8 cells repeatedly stimulated with immature dendritic cells can become $\operatorname{Tr} 1$ cells: regulatory $\mathrm{T}$ cells that produce high levels of IL-10 and moderate amounts of TGF- $\beta$. Importantly, activation of $\mathrm{CD}^{+} \mathrm{T}$ cells in the presence of IL-10 results in the generation of T cell clones that produce IL-10, IFN- $\gamma$, TGF- $\beta$, and IL-5, which have inhibitory effects on autologous naive $\mathrm{T}$ cells.

\section{Control of the specificity of regulatory $\mathrm{T}$ cells}

The identification of subsets of regulatory $\mathrm{T}$ cells has provided a mechanism for immune modulation, but an important, as-yet-unanswered question has been whether the regulatory cells are antigen specific and how specificity is maintained. Uncontrolled nonspecific immune suppression would be as problematic as its absence. In most examples, the effects of the regulatory cells have been demonstrated by their addition to an ongoing immune response. In these models, the immune regulation is not antigen specific but is a feature of the experimental system. For example, the inhibitory effects of $\mathrm{CD} 4{ }^{+} \mathrm{CD} 25^{+} \mathrm{T}$ cells in the NOD mouse are observed because the NOD mouse exhibits an autoimmune response rather than because the regulatory $T$ cells have specificity for diabetogenic T cells (17). Furthermore, soluble factors such as IL-10 or TGF- $\beta$ that are thought to mediate immune regulation are not necessarily specific in their targets. Therefore some means of localizing the regulatory activity to the sites of antigen recognition are needed.

The observations in the report by Arif et al. (18) in this issue of the JCI suggest a mechanism for specificity of immune regulation. The authors identified a panel of peptides from IA-2 and proinsulin that is naturally processed and presented by human leukocyte antigen-DR4 (HLA-DR4). Their aim was to identify epitopes unique to proinsulin, which is the only autoantigen that, outside of the thymus, is localized exclusively to $\beta$ cells. The IFN- $\gamma$ responses to combined proinsulin and IA-2 peptides allowed the researchers to discriminate between T1DM patients and control subjects. An IFN- $\gamma$ response to at least one peptide was observed in $72 \%$ of T1DM patients, compared with

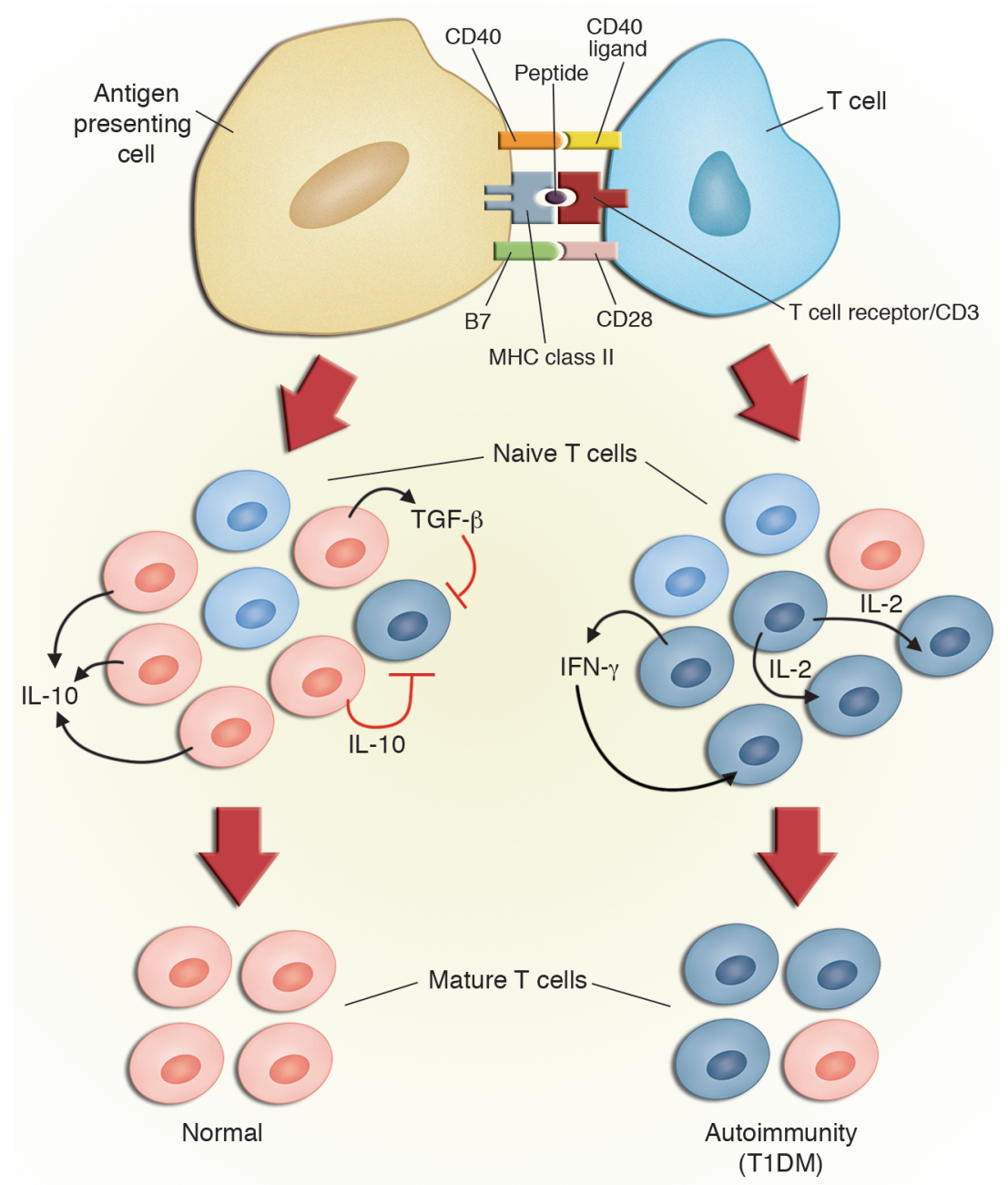

\section{Figure 1}

Immune regulation at the site of antigen presentation. The findings of Arif et al. (18) suggest that the same endogenous peptides activate T cells in patients with T1DM and normal nondiabetic individuals. However, the pathways of differentiation in patients in whom autoimmunity develops and normal individuals in whom autoimmunity is arrested are different. Following engagement of the TCR with antigen in the context of (in the case of CD4 ${ }^{+} \mathrm{T}$ cells) MHC class II and costimulatory molecules, naive T cells (light blue) from normal individuals differentiate into cells characterized by production of IL-10 (light red). Because of the immune regulatory properties of IL-10 as well as other cytokines that may be made with it (such as TGF- $\beta$ ), the cells that mediate an autoaggressive immune response (dark blue) are inhibited at the site of antigen presentation. However, the findings of Arif et al. suggest that in patients with autoimmune diabetes, there is instead induction of a predominant number of $\mathrm{T}$ cells that produce IFN- $\gamma$ and IL- 2 that characterize the autoaggressive immune response and a small number of IL-10-producing $T$ cells. 
immune regulators in healthy controls and prevent the induction of autoantibodies in vivo is not known, but an implication of this finding is that they do so by imparting local immune regulation through the elaboration of IL-10 (Figure 1). IL-10 is a likely regulatory cytokine and would thus affect the cells recruited to the inflammatory site. The specificity of the immune regulation is imparted by the specificity of the responding $\mathrm{T}$ cell's T cell receptor (TCR).

The findings also imply that a difference between healthy subjects and T1DM patients is not in the repertoire of $T$ cells but is in the pathways of differentiation activated in response to antigen (18). A likely scenario suggested by Cobbold et al. (19) is that sustained but incomplete signaling or altered signaling of $\mathrm{T}$ cells generates $\mathrm{T}$ cells of a regulatory phenotype characterized by production of cytokines that regulate other immune cells, most importantly IL-10 and/or TGF- $\beta$ (TGF- $\beta$ responses were not shown in the study by Arif et al.). Because T cells localize to the site of antigen presentation and are activated there by engagement of the TCR and costimulatory molecules, recruit other $\mathrm{T}$ cells, and affect the differentiation of the recruited cells, these cells obstruct the function of competent Th1 and cytotoxic $T$ cells in a scenario called the "Civil Service" model of immune regulation (19). It is likewise intriguing that individuals with onset of T1DM at older ages had a combination of IL-10 and IFN- $\gamma$ responses to the antigens and as a group have been described to have a slower rate of disease progression than younger patients in whom C-peptide (a byproduct of endogenous insulin production that is released on the same equimolar basis as insulin) is generally undetectable after 5 years of disease. The techniques used by Arif et al. did not allow for assessment of quantitative differences in responses to the antigens, so the full extent of differences in cytokine production between individuals with different rates of disease progression cannot be entirely determined, but the qualitative differences suggest the mechanism described above.

\section{T cell activation pathways may account for activation of regulatory cells}

How and why $\mathrm{T}$ cell activation pathways differ between normal individuals and those with autoimmunity will require careful consideration of the parameters involved in immune activation, including characteristics of antigen-presenting cells, costimulatory signals, features of the antigen-presenting molecules, TCR avidity, and others under investigation. The thymus is not cleared of blame, as TCR affinities for antigen in the cells from normal individuals and patients with T1DM were not compared but may be very different and may account for differences in the responses between patients and normal controls. Other differences in $\mathrm{T}$ cell activation events, as well as differences in antigen presentation, may be involved. Barrat et al. (20) have recently shown that treatment with vitamin D3 and dexamethasone at the time of TCR stimulation induces IL-10-producing regulatory $\mathrm{T}$ cells that exert positive feedback to enhance IL-10 production but reduce the differentiation of Th1 and Th2 T cells and can subsequently inhibit experimental autoimmune encephalomyelitis. We found CD 4 IL- $10^{+} \mathrm{T}$ cells that may have a similar inhibitory function in patients that had been treated with the anti-CD3 monoclonal antibody hOKT3r1(Ala-Ala), which delivers a suboptimal $\mathrm{T}$ cell activation signal (21). In addition, the findings obtained with these particular ubiquitously expressed autoantigens may not be the same with all autoantigens.

\section{Insights for immunotherapy}

These new findings described by Arif et al. (18) suggest an approach for controlling immune responses at the site of antigen presentation. An analogous approach is used by the Epstein Barr virus in which viral IL-10 production enables the virus to evade a host-specific immune response (22). Expression of immune-regulatory cytokines in $\mathrm{T}$ cells that recognize autoantigens and home to sites of antigen presentation is a specific approach that is analogous to the naturally occurring mechanisms described by Arif et al.; techniques to cause such expression are under devel- opment. Thus, these new findings concerning the responses of normal individuals and patients with T1DM to autoantigens shed light on the differences in immune responses between these two groups and the mechanisms of pathogenesis of the disease. The findings suggest ways in which regulation of the autoimmune response occur and offer approaches to immune therapy that may induce immune modulation and ultimately tolerance, the immunologist's "Holy Grail."

1. Diabetes Epidemiology Research International Group. 1988. Geographic patterns of childhood insulin-dependent diabetes mellitus. Diabetes. 37:1113-1119.

2. Hypponen, E., Laara, E., Reunanen, A., Jarvelin, M.R., and Virtanen, S.M. 2001. Intake of vitamin $\mathrm{D}$ and risk of type 1 diabetes: a birth-cohort study. Lancet. 358:1500-1503.

3. Ziegler, A.G., Schmid, S., Huber, D., Hummel, M. and Bonifacio, E. 2003. Early infant feeding and risk of developing type 1 diabetes-associated autoantibodies. JAMA. 290:1721-1728.

4. Norris, J.M., et al. 2003. Timing of initial cereal exposure in infancy and risk of islet autoimmunity. JAMA. 290:1713--1720.

5. Atkinson, M.A., et al. 1994. Cellular immunity to a determinant common to glutamate decarboxylase and coxsackie virus in insulin-dependent diabetes. J. Clin. Invest. 94:2125-2129.

6. Oldstone, M.B., Nerenberg, M., Southern, P., Price, J., and Lewicki, H. 1991. Virus infection triggers insulin-dependent diabetes mellitus in a transgenic model: role of anti-self (virus) immune response. Cell. 65:319-331.

7. Pugliese, A., et al. 1997. The insulin gene is tran scribed in the human thymus and transcription levels correlated with allelic variation at the INS VNTR-IDDM2 susceptibility locus for type 1 diabetes. Nat. Genet. 15:293-297.

8. von Herrath, M.G., Allison, J., Miller, J.F., and Oldstone, M.B. 1995. Focal expression of interleukin-2 does not break unresponsiveness to "self" (viral) antigen expressed in beta cells but enhances development of autoimmune disease (diabetes) after initiation of an anti-self immune response. J. Clin. Invest. 95:477-485.

9. Rabinovitch, A. 1998. An update on cytokines in the pathogenesis of insulin-dependent diabetes mellitus. Diabetes Metab. Rev. 14:129-151.

10. Pakala, S.V., Kurrer, M.O., and Katz, J.D. 1997. T helper 2 (Th2) T cells induce acute pancreatitis and diabetes in immune-compromised nonobese diabetic (NOD) mice. J. Exp. Med. 186:299-306

11. Mueller, R., Bradley, L.M., Krahl, T., and Sarvetnick, N. 1997. Mechanism underlying counterregulation of autoimmune diabetes by IL-4 Immunity. 7:411-418

12. Shevach, E.M. 2000. Regulatory T cells in autoimmunity*. Annu. Rev. Immunol. 18:423-449.

13. Chatenoud, L., Salomon, B., and Bluestone, J.A 2001. Suppressor T cells-they're back and critical for regulation of autoimmunity! Immunol. Rev. 182:149-163.

14. Levings, M.K., et al. 2001. IFN- $\alpha$ and IL-10 induce the differentiation of human type $1 \mathrm{~T}$ regulatory cells. J. Immunol. 166:5530-5539.

15. Groux, H., et al. 1997. A CD4 ${ }^{+}$T-cell subset inhibits antigen-specific T-cell responses and prevents colitis. Nature. 389:737-742.

16. Moore, K.W., de Waal Malefyt, R., Coffman, R.L., and O'Garra, A. 2001. Interleukin-10 and the interleukin-10 receptor. Annu. Rev. Immunol. 19:683-765. 
17. Salomon, B., et al. 2000. B7/CD28 costimulation is essential for the homeostasis of the $\mathrm{CD} 4{ }^{+} \mathrm{CD} 25^{+}$immunoregulatory $\mathrm{T}$ cells that control autoimmune diabetes. Immunity. 12:431-440.

18. Arif, S., et al. 2004. Autoreactive T cell responses show proinflammatory polarization in diabetes but a regulatory phenotype in health. J. Clin. Invest. 113:451-463. doi:10.1172/JCI200419585.
19. Cobbold, S.P., et al. 2003. Regulatory T cells and dendritic cells in transplantation tolerance: molecular markers and mechanisms. Immunol. Rev. 196:109-124.

20. Barrat, F.J., et al. 2002. In vitro generation of interleukin 10 -producing regulatory $\mathrm{CD}^{+} \mathrm{T}$ cells is induced by immunosuppressive drugs and inhibited by T helper type 1(Th1)-and Th2inducing cytokines. J. Exp. Med. 195:603-616.
21. Herold, K.C., et al. 2003. Activation of human T cells by FcR nonbinding anti-CD3 mAb, hOKT3 $\gamma 1$ (Ala-Ala). J. Clin. Invest. 111:409-418. doi:10.1172/JCI200316090.

22. Salek-Ardakani, S., Arrand, J.R., and Mackett, M 2002. Epstein-Barr virus encoded interleukin-10 inhibits HLA-class I, ICAM-1, and B7 expression on human monocytes: implications for immune evasion by EBV. Virology. 304:342-351.

\section{How do mutations in lamins $A$ and $C$ cause disease?}

\author{
Howard J. Worman ${ }^{1}$ and Jean-Claude Courvalin ${ }^{2}$ \\ ${ }^{1}$ Department of Medicine and Department of Anatomy and Cell Biology, \\ College of Physicians and Surgeons, Columbia University, New York, New York, USA \\ ${ }^{2}$ Département de Biologie Cellulaire, Institut Jacques Monod, Université Paris 7, \\ Paris, France
}

\begin{abstract}
Mutations in lamins A and C, nuclear intermediate-filament proteins in nearly all somatic cells, cause a variety of diseases that primarily affect striated muscle, adipocytes, or peripheral nerves or cause features of premature aging. Two new studies (see the related articles beginning on pages 357 and 370) use lamin A/C-deficient mice, which develop striated muscle disease, as a model to investigate pathogenic mechanisms. These reports provide evidence for a stepwise process in which mechanically stressed cells first develop chromatin and nuclear envelope damage and then develop secondary alterations in the transcriptional activation of genes in adaptive and protective pathways.
\end{abstract}

J. Clin. Invest. 113:349-351 (2004). doi:10.1172/JCI200420832.

The nuclear lamina is a meshwork of intermediate filaments, primarily localized on the inner aspect of the inner nuclear membrane, that forms from polymerization of proteins called lamins (1-3). In humans, two genes encode B-type lamins, which are constitutively expressed in somatic cells. LMNA (Lmna in mice) encodes developmentally regulated A-type lamins, including the major somatic cell isoforms lamins A and C (4).

Since 1999, mutations in LMNA have been shown to cause several different inherited diseases (Table 1).

Address correspondence to: Howard J. Worman, Department of Medicine, College of Physicians and Surgeons, Columbia University, 630 West 168th Street, Box 83, New York, New York 10032, USA. Phone: (212) 305-8156; Fax: (212) 305-6443;

E-mail: hjw14@columbia.edu.

Conflict of interest: The authors have declared that no conflict of interest exists.

Nonstandard abbreviations used:

Emery-Dreifuss muscular dystrophy (EDMD); Dunnigan-type familial partial lipodystrophy (FPLD).
Some, such as Emery-Dreifuss muscular dystrophy (EDMD) and Dunnigantype familial partial lipodystrophy (FPLD), are rather tissue-specific (5). Others, such as Hutchinson-Gilford progeria syndrome, which is caused by

\section{Table 1}

Diseases caused by mutations in lamins A and C

Striated muscle diseases (cardiomyopathy with variable skeletal muscle involvement)

Autosomal dominant Emery-Dreifuss muscular dystrophy (no. 181350)

Autosomal recessive Emery-Dreifuss muscular dystrophy (no. 604929)

Cardiomyopathy dilated $1 \mathrm{~A}$ (no. 115200)

Limb-girdle muscular dystrophy type 1B (no. 159001)

Partial lipodystrophy syndromes (with or without developmental abnormalities)

Dunnigan-type familial partial lipodystrophy (no. 151660)

Lipoatrophy with diabetes, hepatic steatosis, hypertrophic cardiomyopathy, and

leukomelanodermic papules (no. 608056)

Mandibuloacral dysplasia (no. 248370)

Peripheral neuropathy

Charcot-Marie-Tooth disorder type 2B1 (no. 605588)

Premature aging syndromes

Hutchinson-Gilford progeria syndrome (no. 176670)

Atypical Werner syndrome (no. 277700 for Werner syndrome)

Additional information and original references can be found within ref. 5 and at the Online Mendelian Inheritance in Man database (OMIM; ref. 19). OMIM entry numbers are given in parentheses. 